\title{
Comprehensive Evaluation of Impacts of Connecting Distributed Generation to the Distribution Network
}

\author{
Wei Jin*, Xuemei Shi*, Fei Ge*, Wei Zhang**, Hongbin Wu ${ }^{\dagger}$ and Chengyuan Zhong*
}

\begin{abstract}
In this paper, we study the various impacts of connecting distributed generation (DG) to the distribution network. The comprehensive evaluation index system (CEIS) of four hierarchies is established, considering economy, reliability and voltage quality, and the calculation methods of different indexes are presented. This paper puts forward an improved triangular fuzzy number analytic hierarchy process (ITFNAHP) to weight the second level indexes (SLI) and the third level indexes (TLI), and calculates the variation coefficient to weight the fourth level indexes (FLI). We calculate the comprehensive weight coefficients based on the weight coefficients of the SLI, TLI and FLI, and then calculate the comprehensive evaluation of satisfaction (CES) of different access schemes. On the basis of the IEEE 33-bus example system, simulations of the calculation methods and the comprehensive evaluation method are carried out under different DG access schemes according to the same total investment cost and the same permeability, respectively, and the simulation results are analyzed and discussed.
\end{abstract}

Keywords: Distribution network, Comprehensive evaluation index system, Improved triangular fuzzy number analytic hierarchy process, Comprehensive weight coefficients, Comprehensive evaluation of satisfaction

\section{Introduction}

With the increasing pressure of environmental protection and energy demand, the development of distributed generation (DG) has become the focus of public attention. The access of DG turns the traditional distribution network into a multiple power distribution network which is covered with medium or small power supplies and load. High penetration of DG causes great changes to the steady-state and dynamic characteristics of the distribution network with DG, and as the access capacity and location of DG are different, the impacts are also different [1-2]. Therefore, it is very important to evaluate the impacts of connecting DG to the distribution network to promote the development and application of DG.

Currently, there are some literatures studying the impacts of connecting DG to the distribution network. The authors of [3] studied the voltage improvement, network losses, environmental improvement and economic benefits of a distribution network with DG. Authors in [4] proposed an approach to evaluate the effects of DGs on distribution network expansion planning from the reliability, uncertainty, and operational viewpoints. The impacts of the reliability of DG on the distribution network were

$\dagger$ Corresponding Author: School of Electrical Engineering and Automation, Hefei University of Technology, Hefei 230009, China. (hfwuhongbin@163.com)

* State Grid Anhui Electric Power Company, Hefei 230061, China

** School of Electrical Engineering and Automation, Hefei University of Technology, Hefei 230009, China.

Received: March 31, 2016 ; Accepted: January 17, 2017 evaluated in [5-8]. In [9], the impacts of network planning, relay protection, and the power quality of DG on distribution network were studied. However, all these studies considered only a part of the impact factors when the DG accessed the distribution network, and the proposed evaluation indexes were not comprehensive enough. There was no comprehensive evaluation of the impacts (including economy, reliability and voltage quality, etc.) of connecting DG to the distribution network. Also, there was no comprehensive method of evaluating the impacts of connecting DG to the distribution network. The process of existed evaluation rarely considered the fuzziness of human judgment, and the process of constructing a judgment matrix is complicated. The method for weighting different evaluation indexes is also imperfect.

Therefore, the impacts of connecting DG to the distribution network should consider multi-attribute indexes for a comprehensive evaluation. This paper uses the Delphi Method to filter the comprehensive evaluation index of the impacts of connecting DG to the distribution network, establishes the hierarchical comprehensive evaluation index system (CEIS), and presents the calculation methods of different indexes. The paper puts forward an improved triangular fuzzy number analytic hierarchy process (ITFNAHP) to weight the second level indexes (SLI) and the third level indexes (TLI) and applies the variation coefficient method to calculate the weight coefficients of the fourth level indexes (FLI), and then obtains the comprehensive weight coefficients. Finally, according to the calculated values of the FLI and the comprehensive 
weight coefficients, it is possible to calculate the comprehensive evaluation of satisfaction (CES) of different access schemes for evaluation according to the same total investment cost and the same permeability.

\section{Comprehensive Evaluation Index System}

In this paper, we use the Delphi Method $[10,11]$ to filter the evaluation indexes of the impacts of connecting DG to the distribution network. The Delphi method is originally developed as a forecasting method which relies on a group of experts who anonymously reply to questionnaires and subsequently receive feedback in the form of a statistical representation of the group response, after which the process repeats itself several times rounds. The goal is to reduce the range of responses and arrive at something closer to expert consensus. Also, the method has been widely adopted in decision-making area. We consult the experts in three rounds, and eventually filter the ultimate evaluation indexes. The evaluation indexes are divided into different levels and groups according to their attributes and the subordinate relations among them. The evaluation indexes for the same attribute are in one group and they belong to the same upper level index, while the different groups have different attributes in the process of grouping. What is more, the evaluation index system should comprehensively reflect the impacts of connecting DG to the distribution network and ensure that the different evaluation indexes reflect the problem relatively independently and do not have too much overlap. Based on the above methods, the CEIS of four hierarchies is established from the top down. Among the CEIS, the first level is the CES, the second level is the SLI, the third level is the TLI and the fourth level is the FLI, as shown in Fig. 1.

The Delphi Method takes full advantage of the experience and knowledge of experts and uses an anonymous approach to enable every expert to make his own judgment independently and to remain unaffected by other complex factors. In that way, the final conclusion is reliable. In this paper, the filtering process of the evaluation index causes the opinions of the experts to converge after the information feedback from two rounds, and the final evaluation index has unity.

The CEIS for economy, reliability and voltage quality is established in this paper by combining the evaluation indexes of the distribution network and considering the impacts of connecting DG to the distribution network. Economy is divided into investment cost and benefit, reliability is divided into system reliability and load point reliability and voltage quality is divided into bus voltage and load point voltage.

\section{Calculation of Evaluation Index}

\subsection{Calculation of the economy index}

In this paper, considering various factors, the impact in terms of economy of connecting DG to the distribution network is divided into investment cost (IC) and benefit. The investment cost contains the initial investment cost, operation and management cost, and pollutant treatment cost. The initial investment of DG is converted into an annual value. The mathematical model of the investment cost of unit capacity (ICUC) is as follows:

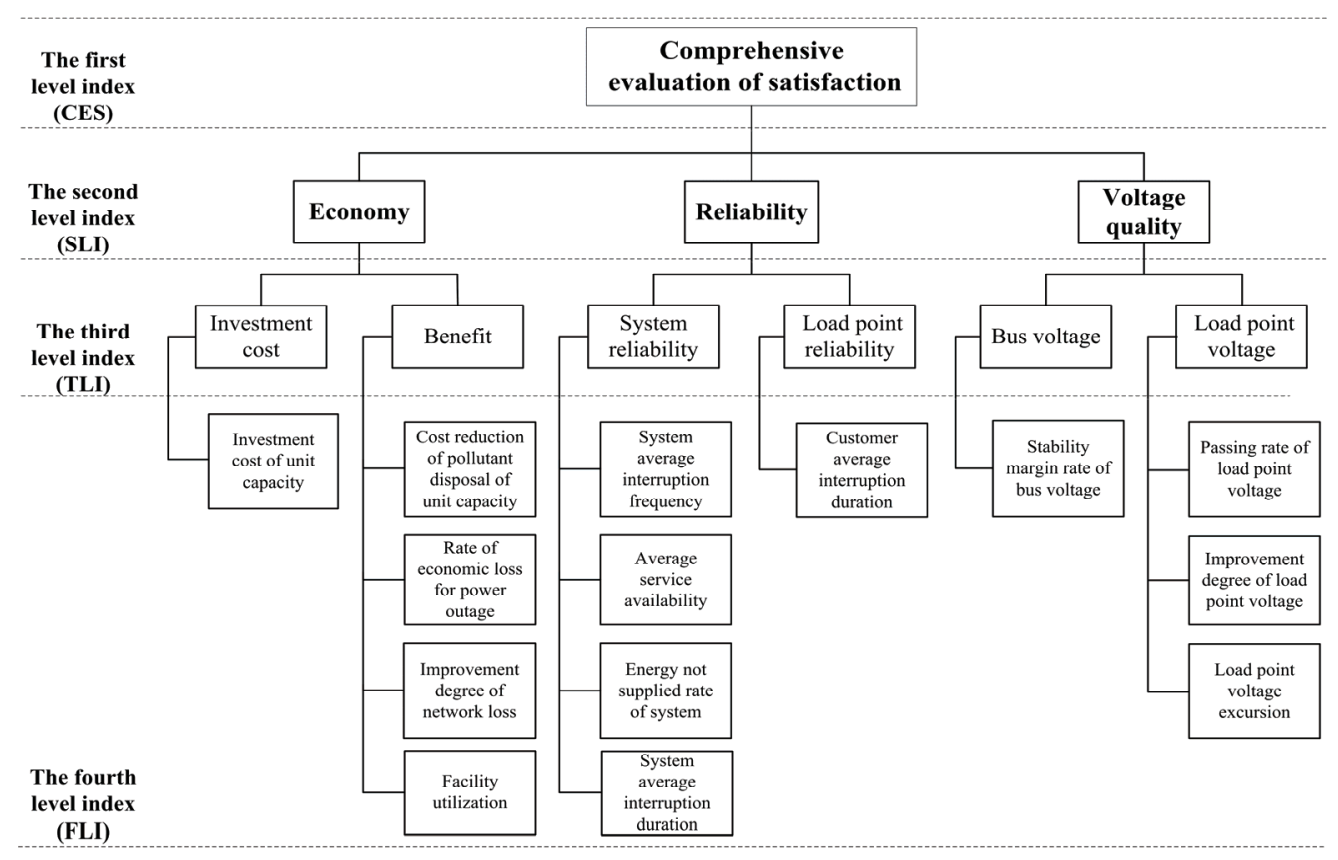

Fig. 1. The comprehensive evaluation index system (CEIS) 


$$
\text { ICUC }=\frac{\sum_{i=1}^{M} N_{i} C_{i} \frac{d(1+d)^{L_{i}}}{(1+d)^{L_{i}}-1}+\int_{0}^{8760}\left(k_{1} \sum_{i=1}^{M} K_{C F i} P_{i}(t)+k_{2} \sum_{i=1}^{M} K_{\text {OMi }} P_{i}(t)\right) d t+\int_{0}^{8760} \sum_{i=1}^{M} C_{k} \gamma_{i k} P_{i}(t) d t}{\int_{0}^{8760} \sum_{i=1}^{M} P_{i}(t) d t}
$$

where $i$ represents different types of DG, $M$ is the species of DG, $N_{i}$ and $C_{i}$ respectively represent the number and unit price of the DG for class $i, d$ is the utilization and $L_{i}$ is the service life of DG for class $i, k_{1}$ and $k_{2}$ are the cost factors, which each take a value of one when considering this cost, and otherwise take a value of zero, $K_{C F i}$ is the coefficient of fuel consumption of DG for class $i, K_{\text {OMi }}$ is the management coefficient of DG for class $i, P_{i}(t)$ is the output power of class $i$ at the moment $t, C_{k}$ is the unit cost of pollutant disposal, and $\gamma_{i k}$ is the pollutant emission coefficient.

In this paper, the benefit consists of the cost reduction of pollutant disposal of unit capacity (CRPDUC), the rate of economic loss for power outage (RELPO), the improvement degree of network loss (IDNL) and facility utilization (FUR).

(i) Cost reduction of pollutant disposal of unit capacity (CRPDUC)

$$
C R P D U C=\frac{C_{w r}-C_{w r}^{\prime}}{P_{D G}}
$$

(ii) Rate of economic loss for power outage (RELPO)

$$
R E L P O=\frac{C_{t d}-C_{t d}^{\prime}}{C_{t d}}
$$

(iii) Improvement degree of network loss (IDNL)

$$
I D N L=\frac{P_{\text {loss }}-P_{\text {loss }}^{\prime}}{P_{\text {loss }}}
$$

(iv) Facility utilization ratio (FUR)

$$
F U R=\frac{P_{D G}}{P_{D G}^{\prime}}
$$

where $C_{w r}$ is the cost of pollutant disposal without DG and $C_{w r}^{\prime}$ is the cost of pollutant disposal with DG, $P_{D G}$ is the output power of DG, $C_{t d}$ is the economic loss for power outage without DG and $C_{t d}^{\prime}$ is the economic loss for power outage with $\mathrm{DG}, P_{\text {loss }}$ is the network loss without DG and $P_{\text {loss }}^{\prime}$ is the network loss with DG, and $P_{D G}^{\prime}$ is the theoretical maximum output power of DG.

\subsection{Calculation of the reliability index}

The reliability in this paper is divided into system reliability and load point reliability. The system reliability consists of system average interruption frequency (SAIF), average service availability (ASA), energy not supplied rate of system (ENSR), system average interruption duration (SAID). And the load point reliability contains customer average interruption duration (CAID).

The improved wind turbine (WT) reliability model is established by combining the Weibull distribution $[12,13]$ with the Markov Chain [14]. Through combining the volatility of light resource with the equipment operation, a multi-state and timing output power model of a photovoltaic (PV) generation system is proposed. The sequential Monte Carlo simulation method $[15,16]$ is adopted to study the reliability evaluation of a distribution network with a WT/PV generation system.

(i) System average interruption frequency (SAIF)

$$
\operatorname{SAIF}=\frac{\lambda_{c}}{N_{y h}}
$$

(ii) Average service availability (ASA)

$$
A S A=\frac{T_{g d}}{T_{n g d}} \times 100 \%
$$

(iii) Energy not supplied rate of system (ENSR)

$$
E N S R=\frac{E N S_{0}-E N S}{E N S_{0}}
$$

(iv) System average interruption duration (SAID)

$$
S A I D=\frac{T_{z}}{N_{y h}}
$$

(v) Customer average interruption duration (CAID)

$$
C A I D=\frac{T_{z}}{\lambda_{c}}
$$

where $\lambda_{c}$ is the customers' total number of power outages, $N_{y h}$ is the total number of customers, $T_{g d}$ is the customers' total hours of power supply, $T_{n g d}$ is the customers' total hours of power supply required, $E N S_{0}$ is energy not supplied without DG, ENS is energy not supplied with DG, $T_{z}$ is the customers' total interruption duration.

\subsection{Calculation of the voltage quality index}

The voltage quality of this paper is divided into bus voltage and load point voltage, including the stability 
margin rate of bus voltage (SMRBV), passing rate of load point voltage (PRLPV), improvement degree of load point voltage (IDLPV) and displacement degree of load point voltage (DDLPV).

(i) Stability margin rate of bus voltage (SMRBV)

$$
S M R B V=\frac{\gamma^{\prime}-\gamma}{\gamma^{\prime}} \times 100 \%
$$

(ii) Passing rate of load point voltage (PRLPV)

$$
P R L P V=\frac{1-t_{c x}}{t_{j c}} \times 100 \%
$$

(iii) Improvement degree of load point voltage (IDLPV)

$$
I D L P V=\frac{\sigma^{\prime}}{\sigma} \times 100 \%
$$

(iv) Load point voltage excursion (LPVE)

$$
L P V E=\frac{\sum_{i=1}^{N_{f h}}\left(U_{i}-U\right)}{N_{f h} \times U} \times 100 \%
$$

where $\gamma^{\prime}$ is the stability margin of bus voltage with DG, $\gamma$ is the stability margin of bus voltage without DG, $t_{c x}$ is the overtime of voltage, $t_{j c}$ is the monitoring time of voltage, $\sigma^{\prime}$ is the load point voltage index with DG, $\sigma$ is the load point voltage index without DG, $N_{f h}$ is the number of load points, $U_{i}$ is the actual voltage for load point $i, U$ is the rated voltage.

Basic electrical operation data can be obtained through the modeling and simulation, making it possible to calculate the evaluation index values under different access schemes according to the above methods.

\section{Comprehensive Evaluation Method}

\subsection{Standardization of the FLI}

There are two kinds of indexes in the CEIS. One is the positive index, for which it is better to have a bigger value. The other is the reverse index, for which it is better to have a smaller value. In order to eliminate the dimension and nature of the index, this paper standardizes the FLI using the Z-score method [17].

The standardization formula is as follows:

$$
\begin{gathered}
x_{i}=\frac{\sum_{j=1}^{m} x_{i j}}{m} \\
s_{i}=\sqrt{\frac{1}{m} \sum_{j=1}^{m}\left(x_{i j}-x_{i}\right)^{2}}
\end{gathered}
$$

$$
z_{i j}=\frac{\left(x_{i j}-x_{i}\right)}{s_{i}}
$$

where $i=1,2, \cdots, n$ and $n$ is the number of the FLI, $j=1,2, \cdots, m$ and $m$ is the number of access schemes, $x_{i j}$ is the actual value of the FLI under different access schemes, $x_{i}$ is the arithmetic average for FLI $i$ and $s_{i}$ is the standard deviation for FLI $i . z_{i j}$ is the standardized FLI value.

Finally, the operational sign of a reverse index should be reversed. After standardization, the index value fluctuates around 0 . If the index value is bigger than 0 , it means that the index value is above average. Otherwise, it means that the index value is below average.

\subsection{Calculation of the weight coefficients}

Filtering the evaluation index inevitably needs human participation, and once the evaluation index is determined, each evaluation index value can be obtained immediately. The results of the comprehensive evaluation depend completely on the weight coefficients. The rationality of weight coefficients which may be calculated objectively or subjectively relates directly to the credibility of the comprehensive evaluation results. The weight coefficient can be calculated by using the objective weighting method or the subjective weighting method, both of which have advantages and disadvantages. The objective weighting method can dispense with any subjective input, but it may lead to the result that different weight coefficients are not uniform on account of different underlying data. The subjective weighting method can keep the criteria consistent for different evaluation objects, but it contains some human factors.

The objective weighting method is adopted to calculate the weight coefficients of the FLI and the subjective weighting method is used to calculate the weight coefficients of the SLI and the TLI in this paper. Based on the weight coefficients of the SLI, TLI and FLI, the comprehensive weight coefficients are calculated, which can reflect the subjective and objective information at the same time. So the comprehensive evaluation method has strong credibility.

\subsubsection{Calculation of the weight coefficients of the FLI}

By analyzing the load flow calculation, reliability evaluation, economic evaluation and voltage quality, it is possible to calculate the FLI values under different access schemes. Due to the different attributes of the FLI, their ranges and dimensions are different. The greater the difference of the index value, the more difficulty to achieve the index. And such index is better at reflecting the gaps among different access schemes. In this paper, we calculate the variation coefficients of the FLI according to the 
arithmetic average and standard deviation of the FLI values under different access schemes, and then the weight coefficients of the FLI can be obtained by the variation coefficients.

Step 1: Calculate the variation coefficients of the FLI

$$
V_{i}=\frac{s_{i}}{x_{i}}
$$

Step 2: Calculate the weight coefficients of the FLI

$$
\omega_{i}=\frac{V_{i}}{\sum_{i=1}^{n} V_{i}}
$$

The weight coefficients determined by the variation coefficient is objective by directly using the data information contained in the FLI to reflect the difference of the evaluation index.

\subsubsection{Calculation of the weight coefficients of the SLI and the TLI}

From the established CEIS of four hierarchies, each TLI includes at least one FLI and each SLI includes two TLI. For the SLI value and the TLI value being calculated from the FLI value indirectly, in order to reflect the subjective and objective information at the same time, the subjective weighting method is adopted in this paper to calculate the weight coefficients of the SLI and the TLI.

The analytic hierarchy process (AHP) $[18,19]$ is often adopted to calculate the subjective weight coefficient, but in the traditional AHP every two elements should be compared with each other once. When there are a large number of elements, this can lead to a large amount of calculation. And the test of judgment matrix [20] consistency is tedious. If it does not meet the consistency requirement, the judgment matrix needs to be reconstructed. The traditional AHP also relies excessively on people's subjective judgment, and does not take into account the judging uncertainty factors. So we put forward the ITFNAHP to calculate the weight coefficient. The steps involved are as follows:

Step 1: Indexes being compared are marked as $a_{1}, a_{2}, \cdots a_{N}$ respectively according to their importance. And $N$ is the number of the index. The rank is $a_{1} \geq a_{2} \geq \cdots \geq a_{\beta} \geq \cdots \geq a_{N}$ according to their importance. Index $\beta$ is compared with index $\beta+1$ and then the corresponding scale value is $t_{\beta}$, where $\beta=1,2 \cdots N-1$. According to the transitivity of the importance to calculate other element values of the judgment matrix, the judgment matrix $R=\left(r_{s v}\right)_{N \times N}$ is established, which has consistency. $r_{s v}$ is the element of the judgment matrix, $s=1,2 \cdots N, v=1,2 \cdots N$. When constructing the judgment matrix, the index number scale method [21] is used, as shown in Table 1.

Step 2: The triangular fuzzy number reciprocal consistent judgment matrix (TFNRCJM) can be constructed by transforming $r_{s v}$ into the form of a triangular fuzzy number $\left(r_{s v}^{l}, r_{s v}^{m}, r_{s v}^{u}\right)$ [22], where $r_{s v}^{m}=r_{s v}, r_{s v}^{l}=r_{s v}^{m}-\frac{r_{s v}^{m}}{N}$, $r_{s v}^{u}=r_{s v}^{m}+\frac{r_{s v}^{m}}{N}, r_{s v}^{m}$ is the likeliest value of the triangular fuzzy number, $r_{s v}^{l}$ is the lower limit value of the triangular fuzzy number, and $r_{s v}^{u}$ is the higher limit value of the triangular fuzzy number.

Step 3: Construction of the lower limit value matrix $A^{l}$, the likeliest value matrix $A^{m}$ and the higher limit value matrix $A^{u}, A^{l}=\left(r_{s v}^{l}\right)_{N \times N}, A^{m}=\left(r_{s v}^{m}\right)_{N \times N}, A^{u}=\left(r_{s v}^{u}\right)_{N \times N}$.

Step 4: Calculation of the normalized eigenvector $y^{l}$ corresponding to the maximum eigenvalue of $A^{l}$, the normalized eigenvector $y^{m}$ corresponding to the maximum eigenvalue of $A^{m}$ and the normalized eigenvector $y^{u}$ corresponding to the maximum eigenvalue of $A^{u}$, $y^{l}=\left(\begin{array}{c}y_{1}^{l} \\ y_{2}^{l} \\ \vdots \\ y_{N}^{l}\end{array}\right), y^{m}=\left(\begin{array}{c}y_{1}^{m} \\ y_{2}^{m} \\ \vdots \\ y_{N}^{m}\end{array}\right), \quad y^{u}=\left(\begin{array}{c}y_{1}^{u} \\ y_{2}^{u} \\ \vdots \\ y_{N}^{u}\end{array}\right)$.

Step 5: Calculation of the intermediate variables $k, h$ and $g$.

$$
\begin{aligned}
& k=\sqrt{\sum_{v=1}^{N} \frac{1}{\sum_{s=1}^{N} r_{s v}^{u}}} \\
& h=\sqrt{\sum_{v=1}^{N} \frac{1}{\sum_{s=1}^{N} r_{s v}^{m}}}
\end{aligned}
$$

Table 1. The meaning of the importance of scale

\begin{tabular}{cl}
\hline Importance of scale & Meaning \\
\hline $9^{0 / 9}$ & The two compared elements are equally important \\
$9^{1 / 9}$ & The former is slightly more important than the latter of the two compared elements \\
$9^{3 / 9}$ & The former is much more important than the latter of the two compared elements \\
$9^{6 / 9}$ & The former is strongly more important than the latter of the two compared elements \\
$9^{9 / 9}$ & The former is extremely more important than the latter of the two compared elements \\
$9^{2 / 9}, 9^{4 / 9}, 9^{5 / 9}, 9^{7 / 9}, 9^{8 / 9}$ & The middle value of above judgment \\
Multiplicative inverse & If the importance of scale is $B_{e f}$ when comparing element $e$ with element $f$, the importance of scale is \\
& $B_{f e}=1 / B_{e f}$ when comparing element $f$ with element $e$
\end{tabular}




$$
g=\sqrt{\sum_{v=1}^{N} \frac{1}{\sum_{s=1}^{N} r_{s v}^{l}}}
$$

Step 6: Calculation of the triangular fuzzy number weight coefficient $w_{s}$ of index $s$.

$$
\begin{gathered}
w_{s}^{l}=k y_{s}^{l} \\
w_{s}^{m}=h y_{s}^{m} \\
w_{s}^{u}=g y_{s}^{u} \\
w_{s}=\left(w_{s}^{l}, w_{s}^{m}, w_{s}^{u}\right)
\end{gathered}
$$

Step 7: Calculation of the weight coefficient $w_{s}^{*}$ of index $S$.

$$
w_{s}^{*}=\frac{1}{2}\left[(1-\alpha) w_{s}^{l}+w_{s}^{m}+\alpha w_{s}^{u}\right]
$$

Where $\alpha$ is the risk factor, which reflects the risk attitude of decision-makers. If $\alpha>0.5$, it means that the decision-makers are pursuing risk. If $\alpha=0.5$, it means that the decision-makers are risk-neutral. If $\alpha<0.5$, it means that the decision-makers are risk-averse.

When the compared indexes are SLI, $N$ is equal to 3. At this time, it constructs a 3 order TFNRCJM based on the economy, reliability and voltage quality according to the ITFNAHP. And $w_{s}^{*}$ is the weight coefficient of the SLI, which is marked as $\lambda_{p}, p=1,2,3$.

When the compared indexes are TLI, $N$ is equal to 2. At this time, it constructs three 2 order TFNRCJMs. The first 2 order TFNRCJM is based on investment cost and benefit, the second 2 order TFNRCJM is based on system reliability and load point reliability and the third 2 order TFNRCJM is based on bus voltage and load point voltage. And $w_{s}^{*}$ is the weight coefficient of the TLI, which is marked as $\eta_{q}, q=1,2,3,4,5,6$.

According to the ITFNAHP, the constructed judgment matrix has strict consistency, which can avoid the tedious process of consistency testing. At the same time, it reduces the workload and takes into account the fuzziness of human judgment. So the ITFNAHP avoids the limitation of the traditional method and can be fully aware of many complex factors.

\subsubsection{Calculation of comprehensive weight coefficients}

According to formula (28), the comprehensive weight coefficient $W_{i}^{*}$ of the FLI with relation to the CES can be calculated based on the weight coefficients of the SLI, TLI and FLI whose values are $\lambda_{p}, \eta_{q}$ and $\omega_{i}$ respectively:

$$
W_{i}^{*}=\frac{\sum_{p=1}^{3} \sum_{q=1}^{6} \zeta \omega_{i} \eta_{q} \lambda_{p}}{\sum_{i=1}^{n} \sum_{p=1}^{3} \sum_{q=1}^{6} \zeta \omega_{i} \eta_{q} \lambda_{p}}
$$

The comprehensive weight coefficient takes into account the objective and subjective factors, as well as the fuzziness of human judgment, which avoids the shortage of data information of the pure objective analysis and the subjective randomness of the pure subjective analysis.

\subsection{Calculation of the CES of different access schemes}

The formula of the CES is as follows:

$$
S_{j}=\sum_{i=1}^{n} W_{i}^{*} z_{i j}
$$

According to formula (29), the CES of different access schemes is calculated, and the one with the biggest CES is the best access scheme.

\section{Analysis of Example}

\subsection{Introduction of the example}

With the IEEE 33-bus example system [23] (as shown in Fig. 2), 7 access schemes are established based on the difference of type, location and capacity of DG. The reference power is $10 \mathrm{MW}$, the reference voltage is 12.66 $\mathrm{kV}$ and the outage rate of lines is 0.046 time/(year $\mathrm{km})$. One WT costs 3.72 million yuan and the rated power is 300 $\mathrm{kW}$, while one PV costs 2.64 million yuan and the rated power is $300 \mathrm{~kW}$. The operation and maintenance coefficient of WT is $0.0296 ¥ / \mathrm{kW}$, while PV is 0.0096 $¥ / \mathrm{kW}$. The power temperature coefficient is -0.0047 and the pollutant disposal coefficient of the distribution network is $0.264 ¥ / \mathrm{kW}$.

In the example system, WT and PV are connected to the end nodes and their output is preferentially used to meet the load demand of the end nodes. Besides, the DGs capacity is less than the load demand of the nodes where they are connected. Thus the example system is a unidirectional flow system and DGs won't output power to

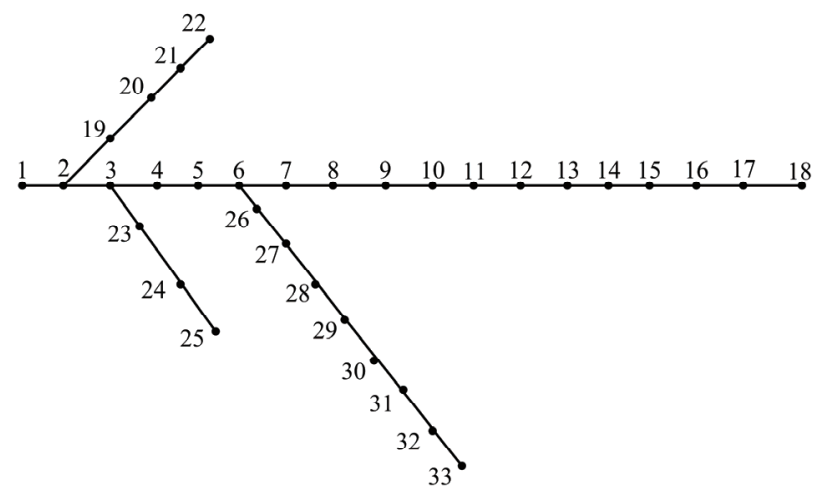

Fig. 2. IEEE 33-bus example system 
the grid.

\subsection{The comparative evaluation under the same total investment cost}

The 7 access schemes under the same total investment cost are shown as follows:

Case 1: The WT costing 1 million yuan is connected to node 18 and the PV costing 4 million yuan is connected to node 25.

Case 2: The WT costing 2 million yuan is connected to node 18 and the PV costing 3 million yuan is connected to node 25 .

Case 3: The WT costing 3 million yuan is connected to node 18 and the PV costing 2 million yuan is connected to node 25.

Case 4: The WT costing 4 million yuan is connected to node 18 and the PV costing 1 million yuan is connected to node 25.

Case 5: The WT costing 5 million yuan is connected to node 18 and without PV.

Case 6: The PV costing 5 million yuan is connected to node 25 and without WT.

Case 7: The WT costing 2.5 million yuan is connected to node 18 and the PV costing 2.5 million yuan is connected to node 25 .

The FLI values are calculated under different access schemes and the results are shown in Table 2. From Table 2, the established CEIS can respond to the impacts of connecting DG to the distribution network from different aspects, and the extent of that impacts vary with the type and investment cost of the DG. According to formulas (15), (16) and (17), the standardized FLI values are calculated and the results are shown in Table 3.

According to the standardized FLI values of different access schemes, a radar chart can be drawn as shown in Fig. 3.

Fig. 3 shows vividly and intuitively the advantages and disadvantages of different access schemes in comprehensive evaluation. Case 6 is superior to the other schemes in ICUC and LPVE, but it has a great shortage in ASA, ENSR and SAID. In the meantime, most of the indexes in Case 7 are higher than the average value. In order to analyze comprehensively the impacts of different indexes, these indexes are weighted and then the CES of different access schemes is calculated.

The variation coefficient method is adopted to weight

Table 2. FLI values of different access schemes under the same total investment cost.

\begin{tabular}{|c|c|c|c|c|c|c|c|}
\hline \multirow{2}{*}{ FLI } & \multicolumn{7}{|c|}{ Access schemes of DG } \\
\hline & Case 1 & Case 2 & Case 3 & Case 4 & Case 5 & Case 6 & Case 7 \\
\hline ICUC & 0.175 & 0.164 & 0.240 & 0.224 & 0.245 & 0.164 & 0.198 \\
\hline$C R P D U C$ & 0.264 & 0.368 & 0.428 & 0.557 & 0.661 & 0.265 & 0.428 \\
\hline RELPO & 0.512 & 0.502 & 0.510 & 0.488 & 0.486 & 0.497 & 0.505 \\
\hline$I D N L$ & 0.088 & 0.150 & 0.105 & 0.125 & 0.120 & 0.089 & 0.127 \\
\hline FUR & 0.670 & 0.819 & 0.556 & 0.667 & 0.667 & 0.661 & 0.665 \\
\hline SAIF & 2.347 & 2.358 & 2.372 & 2.388 & 2.403 & 2.485 & 2.362 \\
\hline$A S A$ & $99.864 \%$ & $99.864 \%$ & $99.864 \%$ & $99.864 \%$ & $99.864 \%$ & $99.856 \%$ & $99.864 \%$ \\
\hline ENSR & 0.058 & 0.055 & 0.054 & 0.057 & 0.056 & 0.025 & 0.057 \\
\hline$S A I D$ & 11.914 & 11.929 & 11.918 & 11.894 & 11.910 & 12.605 & 11.896 \\
\hline$C A I D$ & 5.077 & 5.060 & 5.026 & 4.980 & 4.957 & 5.072 & 5.036 \\
\hline$S M R B V$ & 0 & 0 & 0 & 0 & 0 & 0 & 0 \\
\hline$P R L P V$ & 1 & 1 & 1 & 1 & 1 & 1 & 1 \\
\hline$I D L P V$ & $100.042 \%$ & $100.051 \%$ & $100.096 \%$ & $100.102 \%$ & $100.036 \%$ & $100.045 \%$ & $100.123 \%$ \\
\hline$L P V E$ & $1.437 \%$ & $1.442 \%$ & $1.338 \%$ & $1.360 \%$ & $1.253 \%$ & $1.191 \%$ & $1.236 \%$ \\
\hline
\end{tabular}

Table 3. Standardized FLI values of different access schemes under the same total investment cost

\begin{tabular}{|c|c|c|c|c|c|c|c|}
\hline \multirow{2}{*}{ FLI } & \multicolumn{7}{|c|}{ Access schemes of DG } \\
\hline & Case 1 & Case 2 & Case 3 & Case 4 & Case 5 & Case 6 & Case 7 \\
\hline$I C U C$ & 0.812 & 1.151 & -1.186 & -0.694 & -1.339 & 1.151 & 1.105 \\
\hline$C R P D U C$ & -1.187 & -0.417 & 0.026 & 0.981 & 1.750 & -1.179 & 0.026 \\
\hline$R E L P O$ & 1.273 & 0.212 & 1.061 & -1.273 & -1.485 & -0.318 & 0.530 \\
\hline$I D N L$ & -1.297 & 1.698 & -0.476 & 0.490 & 0.248 & -1.249 & 0.587 \\
\hline$F U R$ & -0.030 & 2.068 & -1.636 & -0.072 & -0.072 & -0.157 & -0.101 \\
\hline SAIF & 0.943 & 0.689 & 0.366 & -0.003 & -0.349 & -2.242 & 0.597 \\
\hline$A S A$ & 0.408 & 0.408 & 0.408 & 0.408 & 0.408 & -2.449 & 0.408 \\
\hline ENSR & 0.572 & 0.299 & 0.208 & 1.482 & 0.390 & -2.434 & 0.482 \\
\hline$S A I D$ & 0.392 & 0.330 & 0.376 & 0.474 & 0.408 & -2.447 & 0.466 \\
\hline$C A I D$ & -1.108 & -0.710 & 0.087 & 1.165 & 1.704 & -0.991 & -0.147 \\
\hline$S M R B V$ & 0 & 0 & 0 & 0 & 0 & 0 & 0 \\
\hline$P R L P V$ & 0 & 0 & 0 & 0 & 0 & 0 & 0 \\
\hline$I D L P V$ & -0.881 & -0.605 & 0.776 & 0.960 & -1.065 & -0.789 & 1.605 \\
\hline$L P V E$ & -1.223 & -1.333 & -0.125 & -0.455 & 0.753 & 1.411 & 0.972 \\
\hline
\end{tabular}


the FLI, and the weight coefficient vector of the FLI is $(0.145,0.285,0.017,0.162,0.095,0.016,0.001,0.190$, $0.018,0.008,0,0,0.001,0.062)$ according to formulas (18) and (19).

The ITFNAHP is adopted to weight the SLI and the TLI and is equal to 0.5 . The weight coefficient vector of the SLI is $(0.217,0.443,0.340)$ and the TLI is $(0.457,0.543$, $0.602,0.398,0.543,0.457)$ according to formulas $(20)$ (27).

According to formula (28), the comprehensive weight coefficient vector of the FLI with relation to the CES is $(0.095,0.222,0.013,0.126,0.074,0.028,0.001,0.335$, $0.032,0.009,0,0,0.001,0.064)$ based on the weight coefficients of the SLI, TLI and FLI.

Lastly, the CES of different access schemes can be obtained according to formula (29), as shown in Fig. 4. As can be seen in Fig. 4, the CES of Case 6 accessing PV is lowest. On the contrary, Case 5 accessing WT obtains the highest CES. By contrast, accessing WT can improve the CES.

Synthesize Fig. 3 and Fig. 4, compared with other cases,
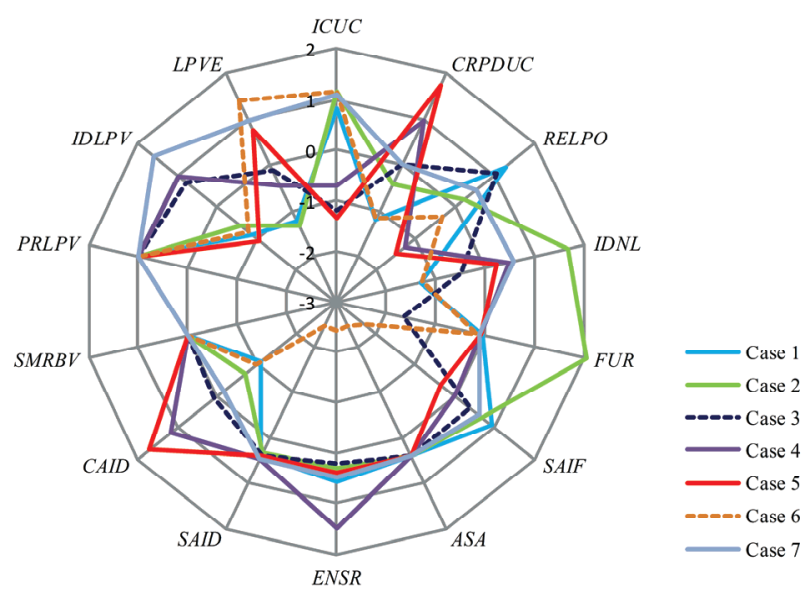

Fig. 3. Radar chart of the standardized FLI values of different access schemes under the same investment cost
Case 5 has obvious advantages in ICUC, FUR and SAIF, which have the biggest comprehensive weight coefficients. So Case 5 has the highest CES, and it is the best scheme. Most FLI values of Case 6 are below averages. Therefore, compared with other cases, there is a great shortage in Case 6 and the CES of Case 6 is also lowest. Case 2 is superior to the other cases in IDNL and FUR, but has a certain advantage in CRPDUC, ENSR, CAID and LPVE. Synthesizing the multiple assessment indexes and weight coefficients of each index, the final calculation of the CES is smaller than best scheme slightly.

\subsection{The comparative evaluation under the same permeability}

The 7 access schemes under the same permeability are shown as follows:

Case 1: The WT of $100 \mathrm{~kW}$ is connected to node 18 and the PV of $400 \mathrm{~kW}$ is connected to node 25.

Case 2: The WT of $200 \mathrm{~kW}$ is connected to node 18 and the PV of $300 \mathrm{~kW}$ is connected to node 25 .

Case 3: The WT of $300 \mathrm{~kW}$ is connected to node 18 and the PV of $200 \mathrm{~kW}$ is connected to node 25 .

Case 4: The WT of $400 \mathrm{~kW}$ is connected to node 18 and the PV of $100 \mathrm{~kW}$ is connected to node 25 .

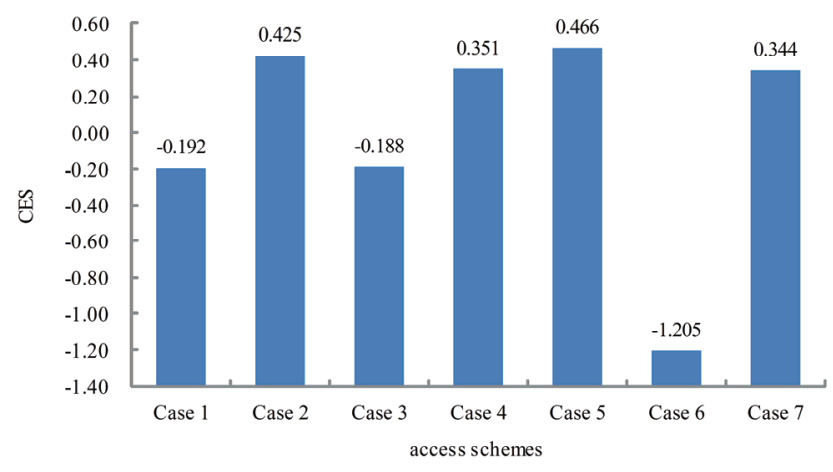

Fig. 4. Bar graph of the CES of different access schemes under the same total investment cost

Table 4. FLI values of different access schemes under the same permeability

\begin{tabular}{|c|c|c|c|c|c|c|c|}
\hline \multirow{2}{*}{ FLI } & \multicolumn{7}{|c|}{ Access schemes of DG } \\
\hline & Case 1 & Case 2 & Case 3 & Case 4 & Case 5 & Case 6 & Case 7 \\
\hline ICUC & 0.214 & 0.220 & 0.188 & 0.280 & 0.246 & 0.166 & 0.335 \\
\hline$C R P D U C$ & 0.358 & 0.355 & 0.462 & 0.564 & 0.660 & 0.264 & 0.264 \\
\hline RELPO & 0.476 & 0.483 & 0.512 & 0.521 & 0.483 & 0.496 & 0.508 \\
\hline$I D N L$ & 0.094 & 0.098 & 0.158 & 0.117 & 0.133 & 0.094 & 0.044 \\
\hline FUR & 0.560 & 0.582 & 0.802 & 0.530 & 0.668 & 0.652 & 0.448 \\
\hline SAIF & 2.349 & 2.368 & 2.382 & 2.401 & 2.417 & 2.485 & 2.376 \\
\hline$A S A$ & $99.864 \%$ & $99.864 \%$ & $99.865 \%$ & $99.864 \%$ & $99.864 \%$ & $99.856 \%$ & $99.864 \%$ \\
\hline ENSR & 0.055 & 0.052 & 0.059 & 0.057 & 0.055 & 0.024 & 0.058 \\
\hline$S A I D$ & 11.943 & 11.949 & 11.866 & 11.896 & 11.931 & 12.611 & 11.882 \\
\hline$C A I D$ & 5.084 & 5.047 & 4.982 & 4.954 & 4.936 & 5.075 & 5.002 \\
\hline$S M R B V$ & 0 & 0 & 0 & 0 & 0 & 0 & 0 \\
\hline$P R L P V$ & 1 & 1 & 1 & 1 & 1 & 1 & 1 \\
\hline$I D L P V$ & $100.081 \%$ & $100.182 \%$ & $100.150 \%$ & $100.104 \%$ & $100.065 \%$ & $100.098 \%$ & $100.108 \%$ \\
\hline$L P V E$ & $1.352 \%$ & $1.381 \%$ & $1.246 \%$ & $1.275 \%$ & $1.358 \%$ & $1.320 \%$ & $1.341 \%$ \\
\hline
\end{tabular}


Table 5. Standardized FLI values of different access schemes under the same permeability

\begin{tabular}{|c|c|c|c|c|c|c|c|}
\hline \multirow{2}{*}{ FLI } & \multicolumn{7}{|c|}{ Access schemes of DG } \\
\hline & Case 1 & Case 2 & Case 3 & Case 4 & Case 5 & Case 6 & Case 7 \\
\hline ICUC & 0.406 & 0.293 & 0.895 & -0.836 & -0.196 & 1.309 & -1.870 \\
\hline CRPDUC & -0.430 & -0.451 & 0.314 & 1.043 & 1.730 & -1.102 & -1.102 \\
\hline$R E L P O$ & -1.326 & -0.884 & 0.947 & 1.515 & -0.884 & -0.063 & 0.695 \\
\hline$I D N L$ & -0.344 & -0.223 & 1.580 & 0.348 & 0.829 & -0.344 & -1.846 \\
\hline FUR & -0.436 & -0.228 & 1.859 & -0.721 & 0.588 & 0.436 & -1.499 \\
\hline SAIF & 1.157 & 0.699 & 0.359 & -0.100 & -0.487 & -2.131 & 0.504 \\
\hline$A S A$ & 0.347 & 0.347 & 0.695 & 0.347 & 0.347 & -2.432 & 0.347 \\
\hline ENSR & 0.313 & 0.050 & 0.664 & 0.489 & 0.313 & -2.406 & 0.576 \\
\hline SAID & 0.276 & 0.252 & 0.598 & 0.467 & 0.325 & -2.432 & 0.534 \\
\hline CAID & -1.341 & -0.657 & 0.544 & 1.061 & 1.393 & -1.174 & 0.174 \\
\hline$S M R B V$ & 0 & 0 & 0 & 0 & 0 & 0 & 0 \\
\hline$P R L P V$ & 0 & 0 & 0 & 0 & 0 & 0 & 0 \\
\hline$I D L P V$ & -0.844 & 1.857 & 1.001 & -0.229 & -1.272 & -0.390 & -0.122 \\
\hline$L P V E$ & -0.624 & -1.279 & 1.778 & 1.123 & -0.624 & 0.031 & -0.405 \\
\hline
\end{tabular}

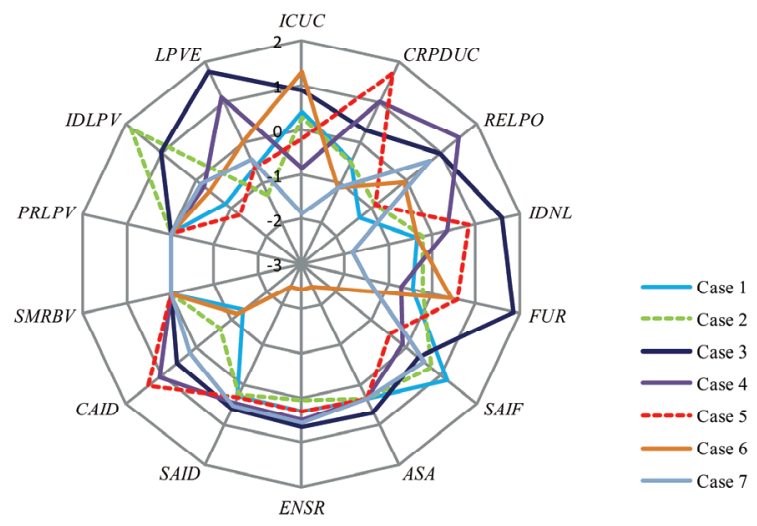

Fig. 5. Radar chart of the standardized FLI values of different access schemes under the same permeability

Case 5: The WT of $500 \mathrm{~kW}$ is connected to node 18 and without PV.

Case 6: The PV of $500 \mathrm{~kW}$ is connected to node 25 and without WT.

Case 7: The WT of $250 \mathrm{~kW}$ is connected to node 18 and the PV of $250 \mathrm{~kW}$ is connected to node 25.

The FLI values are calculated under different access schemes and the results are shown in Table 4.

From Table 4, the impacts of connecting DG to the distribution network vary with the type and permeability of the DG, though the total permeability is constant.

According to formulas (15), (16) and (17), the standardized FLI values of different access schemes are calculated and the results are shown in Table 5.

And then a radar chart can be drawn as shown in Fig. 5. Fig. 5 shows vividly and intuitively the advantages and disadvantages of different access schemes under the same permeability. Case 3 is superior to the other cases in IDNL, FUR, ASA, ENSR and LPVE, but it also reflects some weaknesses on some indexes.

The weight coefficient vector of the FLI is $(0.162,0.241$, $0.023,0.227,0.125,0.012,0.001,0.160,0.015,0.008,0,0$,

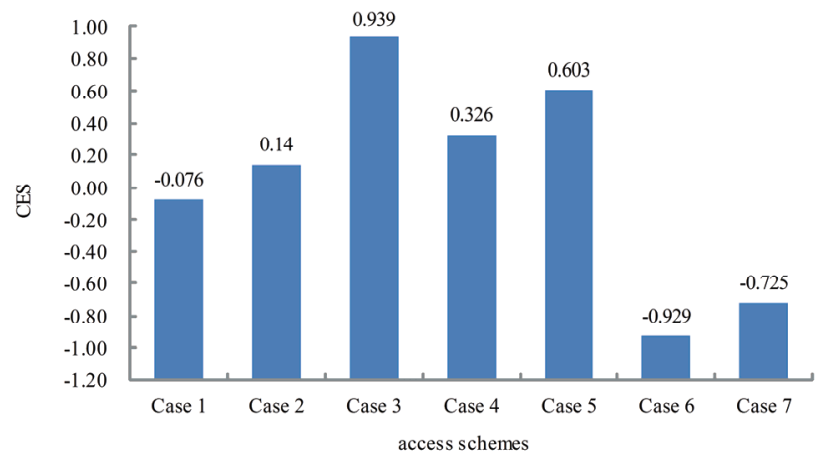

Fig. 6. Bar graph of the CES of different access schemes under the same permeability

$0.001,0.025)$ according to formulas (18) and (19). The weight coefficient vector of the SLI is $(0.217,0.443,0.340)$ and the TLI is $(0.457,0.543,0.602,0.398,0.543,0.457)$ according to formulas $(20) \sim(27)$. Then according to formula (28), the comprehensive weight coefficient vector of the FLI with relation to the CES is $(0.112,0.197,0.019$, $0.186,0.102,0.023,0.001,0.295,0.037,0.009,0,0,0.001$, $0.027)$.

Lastly, the CES of different access schemes can be obtained according to formula (29), as shown in Fig. 6.

Fig. 6 shows visually the comparison of different access schemes in comprehensive evaluation based on the same permeability. Case 3 is the best scheme in the comprehensive evaluation index system established in this paper. The main reason is that Case 3 has a definite advantage in ENSR with the biggest comprehensive weight coefficients, IDNL and FUR with the relatively large comprehensive weight coefficients, compared with other six cases. Column comparison chart shows that the CES of only access PV is lowest and accessing WT can improve the CES. However, if the penetration of WT is too high, the CES will be reduced. Only properly configuring the capacity of PV and WT can get greater CES.

It can be seen that, although the demand is different, the method can determine the best access scheme to meet 
requirements reasonably, by parsing the comprehensive evaluation of the two cases based on the same total investment cost and the same permeability. With the development of distributed generation technology and the in-depth study of the impacts of access to the grid, the comprehensive evaluation system and the evaluation sample both will change. Through the comparisons of comprehensive evaluation for the different demands and different access schemes, this method can provide comprehensive, quantitative information support for power grid enterprises and provide reference for the planning of distributed power grid.

\section{Conclusion}

This paper analyzes the various impacts of connecting DG to the distribution network in terms of economy, reliability and voltage quality. The Delphi Method is used to filter the comprehensive evaluation indexes of the impacts of connecting DG to the distribution network, and the CEIS of four hierarchies is established. The calculation methods of different indexes are also presented and the $\mathrm{Z}$-score method is chosen to standardize the FLI. The ITFNAHP is proposed to weight the SLI and the TLI, which avoids the tedious process of consistency testing and takes into account the uncertainty factors. The variation coefficient is calculated to weight the FLI and the comprehensive weight coefficients are calculated based on the weight coefficients of the SLI, TLI and FLI. So the comprehensive weight coefficients can avoid the shortage of data information from purely objective analysis and the randomness of purely subjective analysis. Finally, all the access schemes are evaluated according to the CES and the one with the biggest CES is the best access scheme according to the same total investment cost and the same permeability, respectively. The proposed comprehensive evaluation method provides a certain decision-making reference for the quantitative assessment of the impacts of connecting DG to the distribution network, and determines the weight coefficients according to the different requirements of planners in order to determine the access capacity and location of the DG. It also provides a basis for scheduling staff to dispatch DG according to the different demands of indexes. Therefore, the proposed comprehensive evaluation method is highly flexible and widely applicable.

\section{Acknowledgements}

This work is supported by the Science and Technology Project of the State Grid Corporation of China (The key technology and empirical research on the coordinated planning of distribution network adapted to the new urbanization).

\section{References}

[1] J. M. Carrasco, L. G. Franquelo, J. T. Bialasiewicz, et al, "Power-electronic systems for the grid integration of renewable energy sources: A survey," IEEE Transactions on Industrial Electronics, vol. 53, no. 4, pp. 1002-1016, Jul. 2006.

[2] M. E. Baran, H. Hooshyar, Z. Shen, et al, "Accommodating high PV penetration on distribution feeders," IEEE Transactions on Smart Grid, vol. 3, no. 2, pp. 1039-1046, Jun. 2012.

[3] P. Chiradeja, R. Ramakumar, "An approach to quantify the technical benefits of distributed generation," IEEE Transactions on Energy Conversion, vol. 19, no. 4, pp. 764-773, Dec. 2004.

[4] B. Amir, M. Hassan, L. Hamid, "Evaluating the effects of renewable and non-renewable DGs on DNEP from the reliability, uncertainty, and operational points of view by employing hybrid GA and OPF," International Transactions on Electrical Energy Systems, vol. 25, no. 12, pp. 3304-3328, Dec. 2014.

[5] Y. M. Atwa, E. F. El-Saadany, "Reliability evaluation for distribution system with renewable distributed generation during islanded mode of operation," IEEE Transactions on Power Systems, vol. 24, no. 2, pp. 572-581, May. 2009.

[6] I. S. Bae, J. O. Kim, "Reliability evaluation of distributed generation based on operation mode," IEEE Transactions on Power Systems, vol. 22, no. 2, pp. 785-790, May. 2007.

[7] B. Liu, Y. Zhang, C. Liu, et al, "Reliability evaluation for distribution systems with distribution generation," European Transactions on Electrical Power, vol. 20, no. 7, pp. 915-926, Oct. 2010.

[8] J. Lin, X. Wang, L. Qin, "Reliability evaluation for the distribution system with distributed generation," International Transactions on Electrical Energy Systems, vol. 21, no. 1, pp. 895-909, Jan. 2011.

[9] B. Yun, Z. Fu, Y. Wang, et al, "The effects of distributed generator accessed to distribution network," International Symposium on Computer, Consumer and Control (IS3C), pp. 146-149, Taichung city, Taiwan, Jun. 2014.

[10] H. A. Linstone, M. Turoff, "The delphi method: Techniques and applications," Addison-Wesley Reading, MA, 2002

[11] M. McGeoch, Y. Brunetto, K. Brown, "The policy delphi method: Contribution to policy and strategy within energy organisations: a 2013 malaysian case study with global implications," Quality \& Quantity, vol. 48, no. 6, pp. 3195-3208, Nov. 2014.

[12] C. D. Lai, M. Xie, D. P. Murthy, "A modified weibull distribution," IEEE Transactions on Reliability, vol. 52, no. 1, pp. 33-37, Feb. 2003.

[13] A. Altunkaynak, T. Erdik, İ. Dabanl, et al, "Theoretical derivation of wind power probability distribution 
function and applications," Applied Energy, vol. 92, no. 4, pp. 809-814, Apr. 2012.

[14] V. V. Lopes, T. Scholz, A. Estanqueiro, et al, "On the use of markov chain models for the analysis of wind power time-series," 11th International Conference on Environment and Electrical Engineering (EEEIC), pp. 770-775, Venice, Italy, May. 2012.

[15] R. Billinton, A. Jonnavithula, "Application of sequential monte carlo simulation to evaluation of distributions of composite system indices," IEE Proceedings Generation Transmission and Distri-bution, vol. 144, no. 2, pp. 87-90, Mar. 1997.

[16] L. B. Fang, J. D. Cai, "Reliability assessment of microgrid using sequential Monte Carlo simulation," Journal of Electronic Science and Technology, vol. 9, no. 1, pp. 31-34, Jun. 2011.

[17] E. Fransen, L. L. Van, N. Lemkens, et al, "A novel zscore-based method to analyze candidate genes for age-related hearing impairment," Ear and hearing, vol. 25, no. 2, pp. 133-141, Apr. 2004.

[18] E. Albayrak, Y. C. Erensal, "Using analytic hierarchy process (AHP) to improve human performance: an application of multiple criteria decision making problem," Journal of Intelligent Manufacturing, vol. 15, no. 4, pp. 491-503, Aug. 2004.

[19] S. I. Ohnishi, T. Yamanoi, H. Imai, "A fuzzy representation for non-additive weights of AHP," IEEE International Conference on Fuzzy Systems (FUZZ), pp. 672-675, Taipei, Taiwan, June. 2011.

[20] Y. C. Wan, B. G. Ma, Z. H. Sheng, "Ranking method for the reciprocal judgment matrix based on the unascertained three-valued judgments," Journal of Systems Engineering and Electronics, vol. 17, no. 1, pp. 115-120, Jan. 2006.

[21] Y. H. Hou, D. J. Shen, "Index Number Scale and Comparison with Other Scales," Systems EngineeringTheory \& Practice, vol. 15, no. 10, pp. 43-46, Oct. 1995.

[22] Y. B. Gong, "A method for priority of triangular fuzzy number reciprocal judgment matrix," IEEE International Conference on Grey Systems and Intelligent Services (GSIS), pp. 942-946, Nanjing, China, Nov. 2007.

[23] A. Zakariazadeh, S. Jadid, P. Siano, "Multi-objective scheduling of electric vehicles in smart distribution system," Energy Conversion and Management, vol. 79, no. 3, pp. 43-53, Mar. 2014.

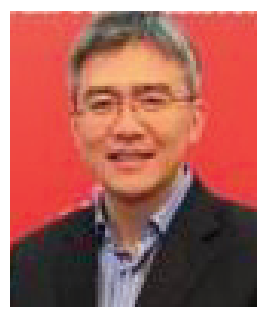

Wei Jin was born in 1970. He is now a senior engineer and vice president of State Grid Anhui Electric Power Company. His research areas include power system planning and source-networkload coordinated control techniques.

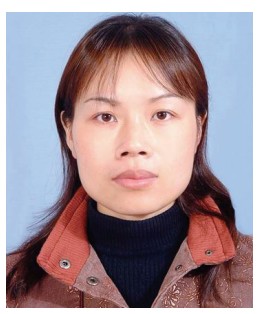

Xuemei Shi was born in 1977. She is now a senior engineer of State Grid Anhui Economic Research Institute. Her research areas include distribution network system planning and design techniques.

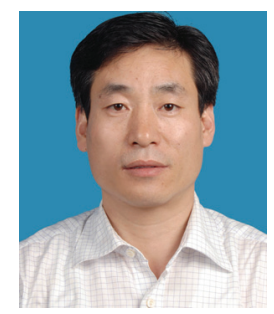

Fei Ge was born in 1972. He is now a senior engineer of State Grid Anhui Electric Power Company. His research areas include power system planning and renewable energy resource techniques.

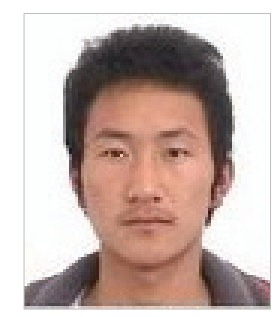

Wei Zhang He received his B.S. degrees in electrical engineering and automation from Hefei University of Technology in 2012. He is now purchasing his M.S. degrees in power system and automation from Hefei University of Technology, Hefei, China. His research interests include distributed power generation technology.

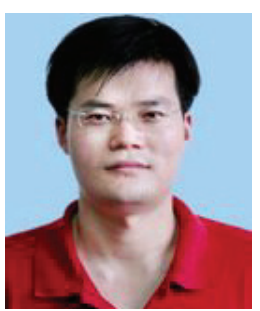

Hongbin Wu He received his B.Sc., M.Sc. and Ph.D. degrees in Electrical Engineering from Hefei University of Technology, Hefei, China in1994, 1998 and 2005 respectively. $\mathrm{He}$ is now a professor of Hefei University of Technology. His research field includes distributed generation technology and distribution network modeling and simulation.

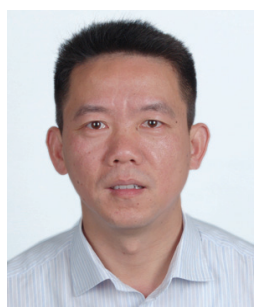

Chengyuan Zhong was born in 1974 . $\mathrm{He}$ is now a senior engineer of State Grid Yuexi Electric Power Company. His research areas include power system operation and control. 